plans for continued engagement around dissemination of results. Accurate measurement of sensitive or stigmatized behavior presents a challenge to the validity of nearly all STI research. Maximizing the acceptability, feasibility and validity of network studies will lead to more accurate estimates of the drivers of STI transmission and will provide more valid insights about the opportunities for interventions to prevent and control STI outbreaks.

Disclosure No significant relationships.

\section{S14.4 THE ROLE OF SEXUAL NETWORKS IN THE GLOBAL SPREAD OF ANTIMICROBIAL-RESISTANCE ENTERIC INFECTIONS}

Nigel Field*. University College London, Centre for Molecular Epidemiology and Translational Research, Institute for Global Health, London, UK

10.1136/sextrans-2019-sti.68

In high-income countries, enteric pathogens have typically affected returning travellers or children, or spread from contaminated point sources. However, sexually transmitted enteric infections (STEI) are now well documented, particularly among men who have sex with men (MSM). The phenomenon was recognised in patients with HIV/AIDS in the early 1970's as arising from direct/indirect ingestion of faecal matter via sexual contact. Bacteria (Shigella spp., Salmonella spp., Campylobacter spp., Escherichia coli), viruses (Hepatitis A), and protozoa (Giardia spp., Cryptosporidium spp., Entamoeba histolytica) have all been implicated. Following a nadir during the 90's and early-2000's, new epidemics of enteric pathogens affecting MSM have been reported internationally. Facilitated by global travel, these have familiar characteristics (oral-anal behaviours and HIV-associations), but also important new features (associations with 'chemsex', social media use, PrEP, syndemic STIs, and transmission in the absence of HIV). For some STEIs, notably Shigellosis, a worrying new feature is resistance to multiple antimicrobial classes found in most isolates from MSM. This may be collateral to frequent antibiotic exposure acting as a selection pressure among the sexual networks affected. Understanding of these sexual networks has been informed by a wide range of approaches, from qualitative patient interviews, through scrutiny of national surveillance trends (male-to-female ratios, gender excess, geographical distributions) and electronic data linkage, to population-level pathogen phylogenomics. These are challenging infections to study, not least due to being sometimes asymptomatic, as well as patient and clinician unawareness about sexual transmission leading to missed or mis-diagnoses, the hidden nature of the affected population, and stigma. Moreover, many questions remain about the prevalence, transmission, duration of infection, clinical implications, drivers of antimicrobial resistance, and effective public health and clinical interventions. Embracing transdisciplinary approaches to understand the sexual networks affected and the behavioural and pathogen-associated drivers seems essential if we are to move from observation to control.

Disclosure No significant relationships.
S15 - NEW APPROACHES TO STI DIAGNOSIS AND PREVENTION

\section{Wednesday, July 17, 2019 10:45 AM - 12:15 PM}

\section{S15.1 FOUR TREPONEMA PALLIDUM PROTEINS DETECTED IN URINE FROM SYPHILIS-INFECTED INDIVIDUALS USING MASS SPECTROMETRY}

Chris Kenyon*. Institute of Tropical Medicine, Berchem, Belgium

\subsection{6/sextrans-2019-sti.69}

Background The direct detection of Treponema pallidum peptides in bodily fluids could facilitate the early diagnosis of initial-, repeat-, congenital- and neuro-syphilis.

Methods To this end, we prospectively recruited 54 individuals with a new diagnosis of syphilis and 6 controls. Their urine specimens were pooled according to disease stage and assessed using complementary mass spectrometry techniques (MALDITOF-TOFMS/MS, LC/ESI-IM-Q-TOF/HDMS ${ }^{\mathrm{E}}$ ) to uncover potential syphilis biomarkers.

Results In total, 26 unique peptides were uncovered corresponding to four unique T. pallidum proteins (Tp0486, Tp0742, Tp0804 and Tp0369) that have no, or minimal, genetic sequence similarity to other known proteins, including prokaryotes and human proteins.

Conclusion This is the first study reporting direct detection of T. pallidum proteins in human biofluid samples using MSbased proteomics methods. These could be promising diagnostic test targets.

Disclosure No significant relationships.

\section{S15.2 CRISPR DIAGNOSTICS: EXPANDING THE NUCLEIC ACID DETECTION TOOLBOX BY HARNESSING MICROBIAL DIVERSITY}

Jonathan Gootenberg*. MIT, Cambridge, USA

\subsection{6/sextrans-2019-sti.70}

Versatile, rapid, and portable sensing of nucleic acids is vital for applications in human health. The RNA-targeting CRISPRassociated enzyme Cas13 has recently been adapted for such purpose. This detection platform, termed SHERLOCK (Specific High Sensitivity Enzymatic Reporter UnLOCKing), can discriminate between inputs that differ by a single nucleotide at very low concentrations and can be lyophilized for portable deployment. However, this technology has several limitations, including the lack of quantitation and reliance on fluorescent detection equipment for readout. Here, we extend the SHERLOCK technology to address these limitations and further develop the utility of this platform. Many applications require detection of more than one target molecule in a single reaction, and we therefore sought to create a multiplex platform that relies on unique cleavage preferences of Cas enzymes. To identify possible candidate enzymes compatible with multiplexing, we biochemically characterized three members of the 